\title{
Ringing the changes: emerging roles for DASH at the kinetochore-microtubule Interface
}

\author{
Graham J. Buttrick • Jonathan B. A. Millar
}

Published online: 27 January 2011

(C) Springer Science+Business Media B.V. 2011

\begin{abstract}
Regulated interaction between kinetochores and the mitotic spindle is essential for the fidelity of chromosome segregation. Potentially deleterious attachments are corrected during prometaphase and metaphase. Correct attachments must persist during anaphase, when spindle-generated forces separate chromosomes to opposite poles. In yeast, the heterodecameric DASH complex plays a vital pole in maintaining this link. In vitro DASH forms both oligomeric patches and rings that can form loadbearing attachments with the tips of polymerising and depolymerising microtubules. In vivo, DASH localises primarily at the kinetochore, and has a role maintaining correct attachment between spindles and chromosomes in both Saccharomyces cerevisiae and Schizosaccharomyces pombe. Recent work has begun to describe how DASH acts alongside other components of the outer kinetochore to create a dynamic, regulated kinetochore-microtubule interface. Here, we review some of the key experiments into DASH function and discuss their implications for the nature of kinetochore-microtubule attachments in yeast and other organisms.
\end{abstract}

Responsible Editors: James Wakefield and Herbert Macgregor

G. J. Buttrick $(\bowtie) \cdot$ J. B. A. Millar $(\bowtie)$

Division of Biomedical Cell Biology,

Warwick Medical School, University of Warwick,

Coventry CV4 7AL, UK

e-mail: g.j.buttrick@warwick.ac.uk

e-mail: j.millar@warwick.ac.uk
Keywords Dam $1 \cdot$ DASH $\cdot$ Kinetochore $\cdot$

Microtubule $\cdot$ Spindle.

$\begin{array}{ll}\text { Abbreviations } \\ \text { CIN } & \text { Chromosomal instability } \\ \text { EM } & \text { Electron microscopy } \\ \text { GDP } & \text { Guanosine diphosphate } \\ \text { GMPCPP } & \text { Guanosine }[(\alpha, \beta) \text {-methyleno]triphosphate } \\ \text { GTP } & \text { Guanosine triphosphate } \\ \text { SPB } & \text { Spindle pole body }\end{array}$

\section{Introduction}

The failure to correctly segregate chromosomes during mitosis is a major cause of chromosomal instability (CIN), the variations in chromosome number that are a hallmark of many solid tumours (Thompson et al. 2010). CIN correlates with poor prognosis in patients and is often associated with increased drug resistance, presumably due to the increased genetic plasticity of the tumour (McClelland et al. 2009).

In normal cells, sister chromatids are faithfully segregated into daughter cells by the mitotic spindle, a microtubule-based organelle that generates the force required for chromosome segregation. Only when all the chromosomes in a cell are correctly bi-oriented (i.e. each sister chromatid is attached, via the spindle, to the opposite pole) are cells allowed to transition from metaphase into anaphase. Constant monitoring and correction of attachments between chromosomes and 
the spindle are required to prevent the formation of aneuploid daughter cells. This interaction between spindle microtubules and chromosomes occurs at kinetochores: large protein structures that assemble on specialised regions of chromatin termed centromeres (reviewed in Przewloka and Glover 2009).

The kinetochore functions as more than just a simple link between chromosomes and the spindle. Not only must the kinetochore efficiently capture highly dynamic microtubules during prometaphase, it must also detect and correct errors in attachment to facilitate bioriented alignment on the metaphase plate. Kinetochore-based signalling pathways generate the spindle assembly checkpoint signal that delays anaphase onset until all chromosomes are correctly oriented. During anaphase A, when sister chromatids are pulled towards opposite poles, kinetochores must be able to transmit the force generated by depolymerising microtubules into movement. The kinetochore is therefore endowed with many intriguing properties that allow efficient and accurate chromosome segregation. For example, micromanipulation experiments in grasshopper spermatocytes show that kinetochore microtubules become more stable when put under tension (Nicklas and Ward 1994). The ability to sense tension between a centromere pair has since been shown to be sufficient for chromosome biorientation in yeast cells (Dewar et al. 2004). In addition, experiments in yeast cells indicate that kinetochores can link microtubule depolymerisation to chromosome movement in the absence of conventional motors (Tanaka et al. 2005; Grishchuk and McIntosh 2006). An understanding of how kinetochores work is essential to our understanding of the process of chromosome segregation.

Almost all of the components of the yeast kinetochore have been discovered, and a similarly complete view is forming of kinetochores in other model systems. Many of the various subcomplexes that make up the kinetochore are conserved from yeast to humans (McAinsh et al. 2003; Liu et al. 2005; Przewloka et al. 2007; Cheeseman and Desai 2008). The exciting challenge ahead is to discover how different kinetochore components act together and with other proteins to generate the dynamic microtubule-chromosome interface. The fungal DASH complex (often called the Dam1 complex, after one of its components) is one kinetochore component that has been subject to intense study in recent years. DASH is a heterodecameric complex that binds microtubules and localises to the outer kinetochore (Nogales and Ramey 2009). Purified Saccharomyces cerevisiae DASH complex has been shown to oligomerise to form rings around microtubules in vitro and can track depolymerising microtubule plus ends (Miranda et al. 2005; Westermann et al. 2005, 2006). Plus-end tracking DASH complexes can couple cargo movement to microtubule depolymerisation, presenting an attractive model by which kinetochores could harness microtubule dynamics to move chromosomes (Asbury et al. 2006; Efremov et al. 2007).

In $S$. cerevisiae cells, the DASH component Dam1 is phosphorylated by Ipl1, an Aurora-related kinase essential for correcting incorrect microtubule-kinetochore attachments (Cheeseman et al. 2002; Pinsky et al. 2006b). This phosphorylation is opposed by the PP1 phosphatase Glc7 and persists in the absence of tension across kinetochores, suggesting that DASH may also be involved in tension-dependent correction of kinetochore-microtubule interactions (Pinsky et al. 2006a; Keating et al. 2009).

A greater understanding of DASH function is rapidly forming through a combination of in vivo and in vitro approaches. In particular, recent advances are beginning to show how DASH functions at the kinetochore and how this function is regulated (Gestaut et al. 2008; Lampert et al. 2010; Tien et al. 2010). Here, we summarise some of the key experiments that have helped establish the role of DASH at the kinetochore and discuss their implications for the nature of the kinetochore-microtubule interface.

\section{The DASH complex}

The first components of the DASH complex were identified in $S$. cerevisiae over 10 years ago. Initial characterisation focused on their localisation to the mitotic spindle and their role in its function (Hofmann et al. 1998; Jones et al. 1999). Further studies demonstrated that the DASH complex localised to the kinetochore and that mutants had severe biorientation defects, leading to the suggestion that DASH may form the link between the kinetochore and spindle microtubules (Cheeseman et al. 2001a,b). Subsequent work steadily increased the number of DASH components identified. We now know DASH to be a complex of ten subunits: Dam1, Duo1, Ask1, Spc34, Spc19, Dad1, 
Dad2/Hsk1, Dad3, Dad4/Hsk2, and Hsk3. Despite the varying sizes of the individual components (Dam1 is almost $40 \mathrm{kDa}$, and Hsk 3 is around $8 \mathrm{kDa}$ ), localisation and function of DASH requires the presence of all subunits, as well as the mitotic spindle (Cheeseman et al. 2001b; Enquist-Newman et al. 2001; Janke et al. 2002; Li et al. 2002).

All components of the DASH complex are conserved in the distantly related fission yeast Schizosaccharomyces pombe. The S. pombe DASH complex was simultaneously discovered through both biochemical and genetic screens (Liu et al. 2005; Sanchez-Perez et al. 2005). Interestingly, whilst all members of the DASH complex are essential in $S$. cerevisiae, DASH is not essential in $S$. pombe. However, DASH mutants display high rates of chromosome missegregation, sensitivity to the microtubule poison thiabendazole and have a long, checkpoint-dependent delay in mitosis (Sanchez-Perez et al. 2005). During anaphase A, poleward movement of kinetochores is much slower in these cells, and some kinetochores frequently 'lag' behind the rest. Severing the spindle by laser ablation reveals that these lagging kinetochores are due to a slower rate of anaphase A, rather than merotelic attachments (Sanchez-Perez et al. 2005; Saitoh et al. 2008; Gao et al. 2010).

\section{Kinetochore capture}

The first crucial role for the kinetochore during mitosis is to allow efficient capture of chromosomes by dynamic spindle microtubules. Tanaka et al. developed an elegant system that allows the regulated inactivation of individual centromeres in $S$. cerevisiae. Cells were blocked in metaphase, and then the centromere was reactivated. Fluorescent markers allowed the tracking of individual kinetochore capture events upon centromere reactivation (Tanaka et al. 2005). Consistent with observations in vertebrate cells, kinetochores initially interact laterally with the wall of the microtubule lattice (Rieder and Alexander 1990; Tanaka et al. 2005). Kinetochores are then rapidly retrieved to the spindle poles before re-orientation on the spindle (Tanaka et al. 2005). Retrieval occurs by one of two mechanisms: 'sliding' along the microtubule lattice, mediated by the kinesin-14 family member Kar3, or DASH-dependent 'pulling' by the depolymerising microtubule. In the presence of Kar3, DASH mutants do not appear to have impaired rates of kinetochore retrieval (Tanaka et al. 2005, 2007).
Conversely, in $S$. pombe, DASH is essential for timely kinetochore retrieval (Franco et al. 2007). Cells harbouring a cold-sensitive allele of the gene encoding $\beta$-tubulin were blocked in a microtubulefree, prometaphase-like state by prolonged incubation at low temperature. Kinetochore capture and retrieval can be monitored upon shift to permissive temperature. Deleting Klp2, the $S$. pombe kinesin-14 has a small effect in population studies using this assay, indicating that motor-mediated sliding plays only a minor role in retrieval in this system (Grishchuk and McIntosh 2006). DASH is absolutely required for retrieval in this assay (Vanoosthuyse et al. 2009). Live imaging of kinetochore recapture is facilitated by deletion of mtol, a gene encoding a centrosomin-like protein that is required for nucleation of cytoplasmic microtubules in $S$. pombe (Sawin et al. 2004). In some $\Delta m t o l$ cells, one centromere pair becomes detached from their characteristic cluster near the spindle pole body (SPB) and must be recaptured by the mitotic spindle before anaphase can progress (Franco et al. 2007). Live imaging of these cells, and cells recapturing kinetochores after microtubule depolymerisation, reveals that deleting either $S$. pombe kinesin-14 only effects kinetochore recapture in a small number of cells (Grishchuk and McIntosh 2006; Franco et al. 2007; Gachet et al. 2008). DASHdeficient cells, however, are severely impaired in these assays, with retrieval only occurring rarely, and after a prolonged delay (Franco et al. 2007; Kobayashi et al. 2007).

This apparent discrepancy in mechanisms of kinetochore retrieval between $S$. cerevisiae and $S$. pombe is perhaps surprising. The extent to which this is due to a fundamental difference between the two species, or differences between the assays used, is unclear. For example, kinetochore capture reported by Tanaka et al. occurs at a greater distance from the SPB than typically seen in the $S$. pombe assays. This 'longer-range' capture may require formation of longer, more stable microtubules better suited to motor-dependent retrieval. Retrieval over shorter distances, as prevalent in the assays used in $S$. pombe, may require shorter and less stable microtubules, better suited to end-on retrieval. Assays in both model systems point to a key role for DASH in kinetochore retrieval and support in vitro experiments that indicate that DASH can couple microtubule depolymerisation to chromosome movement. 


\section{DASH structure and function}

Detailed analysis of the DASH complex structure and function is complicated by the fact that individual DASH components are insoluble when expressed in Escherichia coli (Waldo and Scherrer 2008). However, generation of a polycistronic vector containing the open reading frames (ORFs) of all ten DASH components allowed the purification of complete DASH complexes and was very much the catalyst for the recent insights into DASH function in vitro (Miranda et al. 2005).

The crystal structure of the DASH complex has not yet been solved. However, the architecture of individual DASH complexes has been calculated using electron microscopy (EM) and three-dimensional image reconstruction. This shows DASH to be a bar-shaped complex with a 'bulge' about half-way down its length, from which a protrusion extends. When bound to microtubules, this 'protrusion domain' points towards the microtubule lattice and is likely to be the major site of microtubule attachment on the DASH complex. DASH formed with a truncated form of Dam1 shows a reduced protrusion, indicating that the C-terminus of Dam1 is required for this domain (Wang et al. 2007). Although the precise location of individual subunits within this architecture has yet to be determined, the relative positions of different subunits have been inferred from protein interaction data and structural studies (Nogales and Ramey 2009). In the absence of microtubules, DASH complexes predominately form dimers, with larger oligomers appearing only rarely and in relatively high DASH concentrations (Wang et al. 2007). In the presence of microtubules, however, DASH oligomerises to form rings, and sometimes paired helices, around microtubules (Miranda et al. 2005; Westermann et al. 2005). Careful analysis of negative-stain electron micrographs revealed that oligomers of $16 \mathrm{DASH}$ complexes are required to form rings around microtubules formed of 13 protofilaments (Westermann et al. 2006).

The discovery that DASH forms rings around microtubules captured the imagination of researchers interested in the microtubule-kinetochore interface (Salmon 2005). Rings provide an excellent model for a microtubule plus end tracker that could couple depolymerisation to movement (Efremov et al. 2007; Armond and Turner 2010). A ring-based coupler bound weakly to the plus end would effectively track the depolymerising tip as the splayed microtubule end would push on the ring as the microtubule depolymerises (Fig. 1a). A DASH ring linked to the kinetochore, possibly via the Ndc80 complex [which it interacts with in vitro and in vivo, albeit weakly (Shang et al. 2003)] presents an enticing candidate for such a coupler (Fig. 1a).

DASH is well suited to a role linking the kinetochore to the plus end of the microtubule, due to its observed affinity to guanosine triphosphate (GTP)-tubulin. On microtubules containing stretches of fluorescently labelled GDP-tubulin grown from seeds of unlabelled tubulin assembled with guanosine-5'-[( $\alpha, \beta)$-methyleno]triphosphate (GMPCPP — a stable GTP homologue that is only hydrolysed very slowly), DASH was seen to preferentially bind to the GMPCPP-tubulin seed (Westermann et al. 2005). This indicates that DASH will preferentially associate with GTP-tubulin (as GMPCPP-tubulin mimics GTP-tubulin) and provides a mechanism for DASH association (and potentially kinetochore association) with the microtubule plus end.

Further support for DASH as a coupler came with the observation that DASH tracks the plus end of depolymerising microtubules, as predicted by the model of a ring-based coupler with affinity for the plus end. Microtubules decorated with DASH were depolymerised by reducing the local tubulin concentration to a level that causes microtubule depolymerisation, or by adding the microtubule-depolymerising kinesin-13 XKCM1. Fluorescent labels on the DASH complex and the tubulin allowed DASH to be seen tracking the depolymerising microtubule end (Westermann et al. 2006).

In addition, tip-tracking DASH complexes are capable of transporting cargo in the form of small beads (Asbury et al. 2006; Westermann et al. 2006). This demonstrates that DASH is capable of coupling microtubule depolymerisation to force generation. Optical trapping experiments demonstrate that depolymerising microtubules can move DASH-coated beads under moderate levels of tension. Strikingly, increasing the tension on microtubule-attached DASH beads promotes microtubule growth by reducing the frequency and speed of microtubule depolymerisation events and increasing the frequency of microtubule rescue (Franck et al. 2007). In vivo, chromosome biorientation is ensured by destabilising incorrect kinetochore-microtubule attachments and stabilising 
Fig. 1 Recent models of DASH function. a The properties of DASH in vitro suggest that DASH could form a major microtubulebinding site at the kinetochore, possibly via the Ndc80 complex. Later studies indicate that this is not the case. b DASH localises within the Ndc80 complex, with which it cooperates to facilitate its microtubule association. DASH may form rings or smaller oligomers. c $S$. pombe kinetochores bind two to four microtubules. DASH does not apparently form rings in these cells and appears to favour smaller oligomeric forms. a
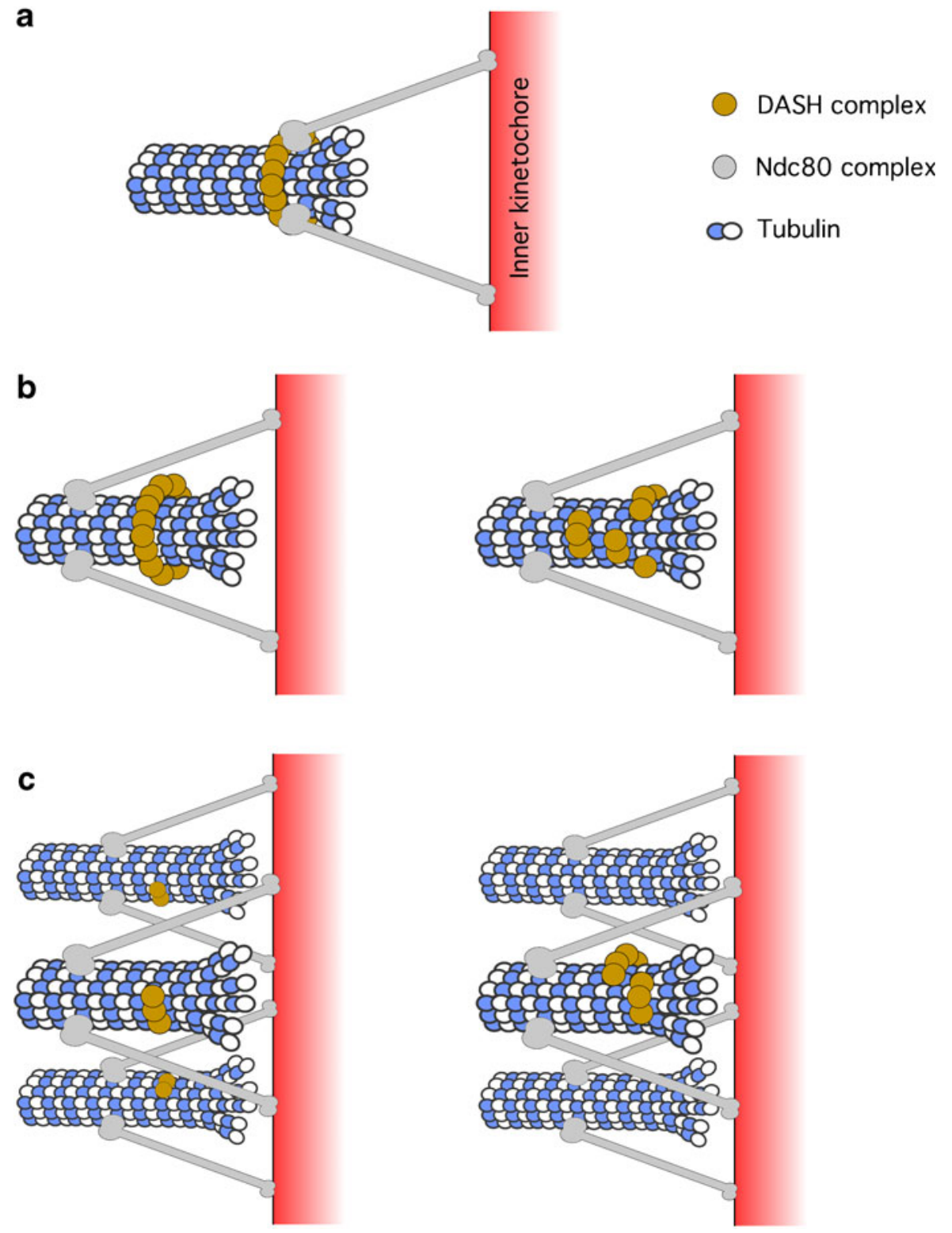

correct, amphitelic, attachments (Tanaka and Desai 2008). Tension across kinetochores is a key indicator of correct attachment. In this assay, DASH bound to beads appears to reconstitute some of the key observed kinetochore behaviours, suggesting that DASH has a role in ensuring correct microtubulekinetochore interactions.

Although the ring-based coupler is undoubtedly an attractive model for DASH function, the presence of rings remains controversial. Crucially, rings have never been seen around microtubules at yeast kinetochores in vivo (McIntosh 2005). The absence of rings may be due to technical difficulties inherent in electron tomography of yeast kinetochores. However, until direct evidence of DASH rings is seen in vivo, the biological relevance of DASH rings remains in doubt.

Interestingly, many of the observed properties of the DASH complex do not require oligomerisation into a ring. Individual GFP-tagged DASH complexes are sufficient to form dynamic attachments to microtubules. Small oligomers corresponding to one to four DASH complexes are also capable of processive tracking of depolymerising plus ends (Gestaut et al. 2008). Non-ring forming DASH complexes also 
appear to be competent to couple microtubule depolymerisation to cargo transport. To observe tip-tracking by smaller DASH oligomers, Grishchuk and colleagues coated microbeads with DASH and observed their tracking on shortening microtubules in the presence or absence of soluble DASH. In the presence of soluble DASH, bead-bound DASH is able to form rings around microtubules. In the absence of soluble DASH, beads prevent the formation of encircling DASH rings. Perhaps surprisingly, DASHcoupled beads still track depolymerising microtubule ends in the absence of soluble DASH, but move more rapidly, and with apparently different kinetics from when DASH is allowed to form rings (Grishchuk et al. 2008).

The existence of two different tip-tracking forms of DASH oligomer in vitro, albeit with different properties, has obvious implications on the nature of the kinetochore-microtubule interface. The physiological relevance of the different oligomerisation states has yet to be determined (Fig. 1b). Potentially, different oligomerisation states could represent DASH at different times during mitosis. The fact that DASH complexes containing Dam1 with mutations on its C-terminus that mimic Ipl1 phosphorylation oligomerise less readily may support this hypothesis (Wang et al. 2007).

\section{DASH at the yeast kinetochore}

Knowing the relative abundance and localisation of DASH at the kinetochore allows us to make some inferences about the likelihood of DASH forming rings physiologically.

Recently, high-resolution microscopy studies by Salmon and colleagues have greatly enhanced the understanding of the composition and architecture of the kinetochore (Joglekar et al. 2006, 2008, 2009). Joglekar et al. were able to take advantage of the ease with which proteins expressed under their endogenous promoter can be fluorescently tagged at their C-termini in yeast. This allowed quantitative assessment of protein number via the relative fluorescent intensity of different tagged proteins. Due to the low protein turnover of kinetochore proteins, Joglekar et al. were able to estimate copy number of different kinetochore components, relative to the $S$. cerevisiae CENP-A homologue, Cse4, in both metaphase and anaphase (Joglekar et al. 2006).
This method estimates that there are 16-20 DASH complexes per kinetochore during metaphase and 10-11 during anaphase-telophase (Joglekar et al. 2006). As each kinetochore binds a single microtubule in $S$. cerevisiae, this indicates 16-20 DASH complexes per microtubule during metaphase. DASH decorates the spindle in anaphase, and this could account for the discrepancy in DASH complex numbers between metaphase and anaphase (Hofmann et al. 1998; Joglekar et al. 2006). These observations are consistent with the model of DASH acting as a ring. There are sufficient quantities of DASH to form rings in metaphase, prior to chromosome segregation. After chromosomes have been separated, during late anaphase and telophase, DASH function at the kinetochore may be less critical, and rings could disperse.

Applying the same technique in $S$. pombe suggests fewer DASH complexes per microtubule (Joglekar et al. 2008). S. pombe centromeres are typically much larger than those in $S$. cerevisiae $(40-100 \mathrm{~kb}$, rather than $150 \mathrm{bp}$ ) and bind two to four microtubules (Ding et al. 1993). Interestingly, the protein composition of an individual microtubule-binding site is largely conserved between $S$. pombe and $S$. cerevisiae, despite the large difference in centromere size between the two species. S. pombe kinetochore protein numbers were estimated at $\mathrm{G} 2 / \mathrm{M}$ transition and in anaphase. During anaphase, there are approximately seven DASH complexes per kinetochore, corresponding to two to three complexes per microtubule (Joglekar et al. 2008). Even if all DASH complexes were clustered onto a single microtubule, there would not be enough to form a single microtubule ring (Fig 1c).

Only one component of the S. pombe DASH complex, Dad1, is present at the kinetochore before entry into mitosis (Sanchez-Perez et al. 2005). During $\mathrm{G} 2 / \mathrm{M}$, there are one to two copies of Dad1 per kinetochore, less than one per microtubule (Joglekar et al. 2008). However, levels of Dad1 appear significantly reduced throughout the cell cycle in the absence of other DASH components (GB, unpublished observations), suggesting that more Dad1 may be recruited to the kinetochore in the presence of other DASH components. Measuring levels of Dad1 at G2/M may therefore be a poor indicator of DASH copy numbers during metaphase. The precise level of DASH at metaphase kinetochores remains to be determined. However, looking at DASH copy numbers during 
anaphase in $S$. pombe, and comparing anaphase and metaphase levels of DASH in $S$. cerevisiae, we can infer that DASH is unlikely to be present in sufficient numbers to form rings around microtubules in $S$. pombe. The most likely form of DASH at S. pombe kinetochores appears to be as small oligomers of two to three complexes, although larger oligomers concentrated on a single microtubule are not ruled out by these observations (Fig. 1c).

Recently, DASH complexes have been seen decorating interphase microtubules in $S$. pombe. In this context, DASH appears to be organised into small 'patches' of two to four complexes (Gao et al. 2010). Interphase patches presumably lack any mitotic regulation that may affect kinetochore DASH. However, the presence of these DASH patches, which move along microtubules and track depolymerising microtubule ends, provides more evidence that DASH oligomers in $S$. pombe do not form rings around microubules (Gao et al. 2010).

\section{Mapping DASH at the kinetochore}

High-resolution fluorescence microscopy has also enabled the creations of a one-dimensional 'map' of the budding yeast kinetochore. Using pairs of proteins fused to enhanced green fluorescent protein and tdTomato (a red fluorescent protein), the relative positions of different kinetochore proteins along the kinetochore-microtubule axis were estimated with up to $10 \mathrm{~nm}$ accuracy. DASH was localised to the outer kinetochore, around $60 \mathrm{~nm}$ away from the Cse4 nucleosome during metaphase. During anaphase, the whole kinetochore contracts, presumably due in part to loss of tension after cohesin degradation. Consistent with this contraction, DASH moves to around $40 \mathrm{~nm}$ away from Cse4, but maintains its position relative to the rest of the kinetochore. Surprisingly, DASH is not the most distal kinetochore component. The microtubule-binding $\mathrm{N}$-terminus of $\mathrm{Ndc} 80$ is consistently $5-10 \mathrm{~nm}$ further from the centromere (Joglekar et al. 2009). The localisation of DASH within an Ndc80 'cage' at the kinetochore has implications for DASH function (Fig 1b). The geometry the Ndc80 complex appears to preclude a DASH ring between the microtubule and $\mathrm{Ndc} 80$. The authors propose that DASH either forms a ring outside the Ndc 80 complex or that smaller oligomers form a 'broken ring' interrupted by $\mathrm{Ndc} 80$ complexes
(Joglekar et al. 2009). Clearly, both these models have different implications for DASH function, and further study is required to determine the correct arrangement of DASH within the kinetochore.

The revelation that DASH is not the most distal component of the kinetochore also suggests a subtler role for DASH at the kinetochore than early models (e.g. Fig. 1a). Localisation within the Ndc80 complex implies that, rather than forming the major kinetochore-microtubule attachment site, DASH has less major role, acting in concert with other kinetochore components.

\section{Cooperation between DASH and Ndc80 complexes}

In higher eukaryotes, a highly conserved network consisting of the Ndc80 and Mis 12 complexes, and the outer kinetochore component Blinkin/Spc105/KNL-1, has been proposed as the major microtubule-binding site in the kinetochore (Cheeseman et al. 2006). Whilst these complexes (termed the $\mathrm{KMN}$ network) are conserved across eukaryotes, DASH homologues have not been found outside fungi (Fig. 3). Recently, purified $S$. cerevisiae $\mathrm{Ndc} 80$ complex has been shown to be sufficient to form load-bearing attachments to microtubule tips in vitro, supporting the assertion that the KMN network is the major linker between kinetochores and microtubules in all eukaryotes (Powers et al. 2009). In S. cerevisiae and, to a lesser extent, $S$. pombe, DASH is essential for correct end-on interaction between microtubules and kinetochores. The precise role of DASH in mediating these interactions has, until recently, been unknown.

Two recent, independent studies demonstrate that the major role of DASH at the kinetochore may be to facilitate microtubule tip-tracking by the Ndc 80 complex (Lampert et al. 2010; Tien et al. 2010). Both studies show, in agreement with previous work, that Ndc80 complex alone forms relatively transient associations with microtubules (Powers et al. 2009; Lampert et al. 2010; Tien et al. 2010). Adding increasing concentrations of DASH reduces the rate of $\mathrm{Ndc} 80$ dissociation from microtubules. In the presence of DASH, $\mathrm{Ndc} 80$ forms prolonged associations with assembling and disassembling microtubule tips (Lampert et al. 2010; Tien et al. 2010). This tip-bound Ndc80 forms load-bearing attachments that can transport beads on both assembling and disassembling microtubules in the presence of DASH. 
By recruiting Ndc80 to microtubules, and facilitating its plus-end tracking, DASH could allow dynamic, regulated attachment between the kinetochore and the microtubule plus end. This model for DASH function explains the observed defects in kinetochore retrieval observed in DASH-deficient yeast cells. It also explains how DASH can be essential for kinetochore function, yet only apparently interact weakly with the rest of the kinetochore. The interaction sites between $\mathrm{Ndc} 80$ and DASH have not yet been characterised. Doing so will show how DASH can help recruit $\mathrm{Ndc} 80$ to microtubules and potentially how this link is regulated.

Whole kinetochores purified from $S$. cerevisiae also exhibit microtubule-binding activity in vitro and can track depolymerising microtubule ends. Intriguingly, these attachments are stabilised when subjected to levels of tension of a similar order to that generated by the mitotic spindle. Purified kinetochores deficient in either Ndc80 or Spc105 show severely impaired microtubule binding. DASH-deficient kinetochores have near wild-type affinity for microtubules, indicating that there are other mechanisms for generating stable kinetochore-microtubule interactions. Functional DASH is still essential to stabilise attachments under tension, however, suggesting that this may be a major role for DASH in vivo (Akiyoshi et al. 2010).

DASH as a template for building a kinetochore 'top-down'

Recent attempts to build a 'minimal' functional kinetochore in yeast provide interesting insights into interaction between DASH and the outer kinetochore. 'Natural' kinetochores are thought to be assembled 'bottom-up' on centromeres; specialised regions of chromatin. In order to establish the minimal kinetochore required for chromosome biorientation, two different groups employed broadly similar techniques to generate artificial kinetochores in S. cerevisiae. Briefly, plasmids were generated that did not contain centromeres, but were artificially tethered to a variety of different kinetochore components and microtubule binding proteins. Tethering components of the inner kinetochore or the KMN network did not produce a functioning kinetochore. Remarkably, artificially tethering DASH component Ask1 to an acentric plasmid not only allows the plasmid to interact with microtubules but also allows it to biorient and segregate with an accuracy approaching that of 'natural' kinetochores (Kiermaier et al. 2009; Lacefield et al. 2009).

Accurate chromosome segregation with an artificial kinetochore requires components of the DASH, $\mathrm{Ndc} 80$ and Mis 12 complexes. This suggests that a DASHbased artificial kinetochore recruits outer kinetochore components, and these components cooperate with DASH to facilitate chromosome biorientaton. The 'top-down' formation of these artificial kinetochores has interesting consequences for kinetochore function. Tethering inner kinetochore components to an acentric plasmid does not support effective biorientation. This means that centromeric DNA is absolutely required for 'bottom-up' building of a working kinetochore. Perhaps, the centromere imposes geometry on the nascent kinetochore that is essential to build a functional kinetochore in this way. Top-down kinetochores do not require the inner kinetochore CBF3 complex (Lacefield et al. 2009), indicating that the major role for this complex is to act as a platform upon which to build the outer kinetochore.

The in vivo significance of a DASH-based artificial kinetochore remains to be seen. However, the observation that DASH can recruit components of the KMN network to form a working kinetochore suggests that DASH may play a key role in organising and regulating the microtubule attachment site at the kinetochore.

\section{Phospho-regulation of DASH}

Timely phosphorylation and dephosphorylation of kinetochore components is essential for correct biorientation. A screen for in vivo phosphorylation sites on different $S$. cerevisiae kinetochore complexes revealed 13 phosphorylation sites on the DASH complex (Cheeseman et al. 2002). Dam1 was shown to be phosphorylated on four sites: one at the N-terminus of the protein and three sites in the C-terminal domain believed to correspond to the 'protrusion domain' (Cheeseman et al. 2002; Wang et al. 2007); Fig. 2). All four of these sites were shown to be targets of the budding yeast Aurora-like kinase, Ipl1. Ipl1 has a role in detecting and correcting incorrect microtubule attachments and is essential for correct biorientation (Biggins et al. 1999; Pinsky et al. 2006b). Changing most of these sites to alanine confers sensitivity to the microtubule poison benomyl and has an additive effect 


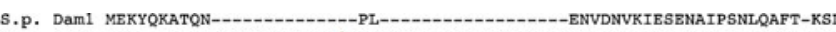
S.c. Dam1 MSE---DKAKLGTTRSATEYRLSIGSAPTSRRSSMGESSSLMKFADQEGLTSSVGEYNENTIQQLLLPK N.s. Dam1 MPDSQTPAPQRSTSRPRTPNRPTTPLRPSSSS--------LRESARESIHGSSASF---PLNAFE-PAF G.z.Dam1 MSMTPKDAAKRPNSRSRTS-RPTTPLRPSSRSS-------FRDSARSARH-HDAPF---PLNAFE-PAF E.n.Dam1 MDAA--S---RSSSRSRAVSRPTTPLRPGSRS--------VREAHGYDRKISRVPYTQPAINALE-POE A. . Dam1 MDPTAFS---RSSSRPRPSSRPTTPLRPSSRS--------LREAHGYGGSISNAGYTQPAINALE-PQF

s.p. Dam1 AVLDDNVSEFRKRMNHLSATKQILDNFNESFSSFLYGLQINAFCVDYENAP--LSESFL----s.c. Dam1 RELSDSIITLDSNFTRLNFIHESLADLNESLGSLLYGIMSNSWCVEFSQAPHDIQDDLIAIKQLKSLEDE N.s.Dam1 AELADAMADLEANMMHFQLMHESLARFSEDFASFLYGLNMENAFCVDFPEGP--LTESFK----------

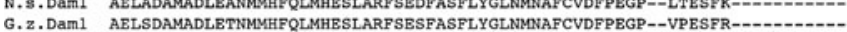
E.n.Dam1 ADLADRMAELEANFDKLQLMHESLTRFSESFASFLYGLNMNAFCVDFPEA---VPESFR-A.०.Dam1 AELADSMADLEANFMHLQLMHESLTRFSESFASFLYGLNMNAFCVDFPEAP--IPESFK-------

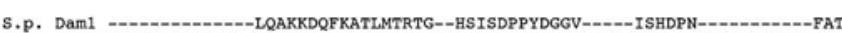
s.c. Dam1 KNILVMELSNMERGIKRKKDEQGENDLAKASQNKQFNQPLFPSSQVRKYRSYDNRDKRKPSKIGNMLQVE N.s. Dam1 --G.z.Dam1 E.n.Dam1 - - Dam1 -

s.p. Dam1 ADETF---ATNDTSFIERPETY-----SAS-S.c. Dam1 NEEDYEDDTSEEASFVLNPTNIGMSKSSQGHVTKTTRLNNNTNSKLLRKSTLHTIRNSTASGADLPIEND N.s.Dan1 GETTF---MTTDTSFVDNPTSN-----ISGPTPRKPLVAPKTPAPRQSRVPAPSG---TTRGT------G.z.Dam1 ---TF---MTTDGSFVENPPT--------TTKTSKFTTPEPPKRQSRLPAAPGRGTTARGRE. D.DAM1 ADMTF---MTSDTTFIBHPPST-----VS------TKQVPK-PGTSRGRDCGNF-KGADGALK--

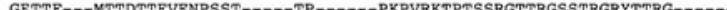

S.p. Dam1 --s.c. Dam1 NVVNLGDLHPNIRISLGSGAARVVIGPVTKMRNSMFSGRAERKPTESRHSVAKKTEKKINTRPPFR N.3.Dam1 - 1 G.z.Dam1 - E.z.Dam1 A. o.Dam1 - -

Fig. 2 Alignment of Dam1 homologues from different fungi. Dam1 from S. pombe, S. cerevisiae, Neurospora crassa, Giberella zeae, Aspergillus nidulans and Aspergillus oryzae. Yellow circles denote Ipl1-dependent phosphorylation sites. Blue circles highlight characterised putative Mps1 sites. Table indicates conservation of

with temperature-sensitive ipll mutants. Changing the same sites to aspartic acid, in an attempt to mimic constitutive phosphorylation, partially rescues the temperature sensitivity of the same ipll mutant (Cheeseman et al. 2002).

Phosphomimetic DASH complexes generated in vitro (hereafter referred to as $4 \mathrm{D} \mathrm{DASH}$ ) show no significant change changes in DASH monomer structure. However, they show a much-reduced tendency to oligomerise into rings around microtubules: At concentrations where oligomeric DASH rings are normally common, 4D DASH typically remains as monomers and dimers (Wang et al. 2007).

DASH complexes phosphorylated by purified Ipl1 also show reduced affinity for microtubules. In this assay, changing the N-terminal Ipl1 site of Dam1 to Alanine (S20A DASH) reversed this effect. Ipl1phosphorylated S20A DASH was indistinguishable from unphosphorylated DASH in its microtubule binding kinetics, demonstrating that phosphorylation of Dam1 at Serine 20 alone regulates microtubule binding (Gestaut et al. 2008).

Ipl1 phosphorylation of Dam1 also regulates the interaction between DASH and the Ndc80 complex. 4D DASH binds less well to Ndc80 than 4A or wildtype DASH in in vitro pull-downs or by yeast twohybrid (Shang et al. 2003). Unphosphorylated S20A

\begin{tabular}{|c|c|c|c|}
\hline S. cerevisiae & S. pombe & $\begin{array}{c}\text { Kinase } \\
\text { (S. cerevisiae) }\end{array}$ & $\begin{array}{l}\text { Regulatory role } \\
\text { (S. cerevisiae) }\end{array}$ \\
\hline $\mathrm{S} 20$ (-RLSIG-) & Not conserved & \multirow{4}{*}{ Ipl1 } & Microtubule binding \\
\hline S257 (-RKSIL-) & Not conserved & & \\
\hline S265 (-RNS IA-) & Not conserved & & $\begin{array}{l}\text { Co-operativity with } \\
\text { Ndc80 complex }\end{array}$ \\
\hline S292 (-RI SLG-) & Not conserved & & \\
\hline $\begin{array}{l}\text { S218 (-TSSEA-) } \\
\text { S221 (-EASFV-) }\end{array}$ & $\begin{array}{l}\text { Not conserved } \\
\text { S143? (-DTS FI -) }\end{array}$ & Mps1 (in vitro) & $\begin{array}{l}\text { Kinetochore } \\
\text { association with } \\
\text { microtubule plus- } \\
\text { end }\end{array}$ \\
\hline
\end{tabular}

Ipl1 and putative Mps1 phosphorylation sites between $S$. cerevisiae and $S$. pombe. The long $\mathrm{C}$-terminus of $S$. cerevisiae Daml is absent in $S$. pombe, and none of the Ipll sites are conserved.

DASH enhances microtubule tip tracking by the Ndc80 complex in vitro. However, Ipl1 phosphorylated S20A DASH does not (Tien et al. 2010). As there is no phosphorylation at Serine 20, DASH phosphorylation by Ipl1 is not regulating DASH association with microtubules in this experiment. Ipl1 phosphorylation of the three $\mathrm{C}$-terminal phosphorylation sites of Dam1 must therefore be regulating the interaction between DASH and Ndc80.

Phosphorylation of Dam1 in $S$. cerevisiae is opposed by the PP1 phosphatase Glc7 (Pinsky et al. 2006a). PP1 phosphatases are targeted to kinetochores and have been shown to regulate chromsome biorientation, congression and spindle checkpoint silencing (Pinsky et al. 2009; Vanoosthuyse and Hardwick 2009; Kim et al. 2010; Liu et al. 2010). Dam1 phosphorylation by Ipl1 requires microtubules, but is reduced in metaphase. In the absence of sister chromatid cohesion, and therefore inter-kinetochore tension, Dam1 phosphorylation persists (Keating et al. 2009). The tension-dependent mechanism of Ipl1dependent phosphorylation at the kinetochore remains unknown. However, Ipl1 phosphorylation of Dam1 presents a useful paradigm for Auorora B-mediated correction mechanisms in other systems.

Interestingly, none of the four Ipll sites in $S$. cerevisiae are conserved in S. pombe. In part, this is 
because $S$. pombe Dam1, along with other fungal Dam1 homologues, lacks the long C-terminal domain of $S$. cerevisiae Dam1 (Fig. 2). Phosphorylation of these sites is implicated in regulating DASH ring formation (Wang et al. 2007), so this may be a consequence of DASH not apparently forming rings in $S$. pombe. Mutations that change, or completely remove, the C-terminal 27 amino acids of $S$. pombe Dam1 confer resistance to thiabendazole (Sanchez-Perez et al. 2005; Griffiths et al. 2008). This suggests that the C-terminus of $S$. pombe Dam1 is important for DASH function. It would be of interest to see whether it represents a major site of DASH regulation, as in $S$. cerevisiae.

$S$. cerevisiae Dam1 is also phosphorylated in vitro by the kinase Mps1 (Shimogawa et al. 2006). Mps1 is required for spindle assembly checkpoint activity as well as SPB duplication in S. cerevisiae (Weiss and Winey 1996). Shimogawa et al. describe in vitro Mps1 phosphorylation of Dam1 at Serines 218 and 221 , which are within a region conserved in S. pombe (Fig. 2), suggesting that this may represent a broadly conserved mechanism for regulating DASH. Mutating these sites to alanine appears to cause kinetochores to cluster nearer spindle poles during metaphase and alters the relative distributions of kinetochores and the microtubule plus end protein Bik1 (the budding yeast CLIP170 homologue). The authors conclude that Mps1 phosphorylation of Dam1 is required to link kinetochores to microtubule plus ends. As DASH appears to preferentially bind the microtubule plus end in the absence of phosphorylation in vitro (Westermann et al. 2005), and there is no direct evidence for Mps1 phosphorylation of Dam1 in vivo, the significance of this observation remains unclear. More work is needed to determine the effect of Mps1 phosphorylation on DASH in vitro, as well as determine its significance in vivo.

The only recorded phosphorylation of an S. pombe DASH component is phosphorylation of T136 in $S$. pombe Ask1 (Wilson-Grady et al. 2008). This threonine is part of a consensus Cdk1 phosphorylation motif. Ask1 is phosphorylated in a cell-cycle specific manner consistent with Cdk1 phosphorylation (Liu et al. 2005). S. cerevisiae Ask1 is also phosphorylated by Cdk1, although phospho-null mutants of these sites have comparatively mild phenotypes, suggesting that this is not an essential mechanism by which DASH is regulated ( $\mathrm{Li}$ and Elledge 2003).
A non-fungal DASH analogue?

Given the observed role of DASH in facilitating microtubule tracking by the yeast kinetochore, and the lethality of DASH deletions in S. cerevisiae, it is perhaps surprising that no direct homologues of the DASH complex have been observed outside of fungi. The other essential microtubule-binding component of the kinetochore, the KMN network, is absolutely conserved from yeast to humans (Fig. 3).

Higher eukaryotes would not need a DASH complex if the KMN network alone were capable of linking kinetochores to dynamic microtubules in a processive manner. Purified C. elegans Ndc80 complex can link cargo to dynamic microtubules; however, much like $S$. cerevisiae $\mathrm{Ndc} 80$, the movement is not very processive (McIntosh et al. 2008). This suggests that the Ndc80 complex in higher eukaryotes still requires cofactors to allow processive interaction with microtubules. The need would be even more pressing in large cells such as human cells, given their size relative to the small yeast nucleus.

Electron tomography of microtubule ends at kinetochores in PtK1 cells reveals fine 'fibrils' that appear to extend from the inner kinetochore to the inside edge of splayed microtubule protofilaments. These kinetochore fibrils, which have not been reported in yeast, could facilitate attachment of dynamic microtubules to the kinetochore (McIntosh et al. 2008). In the absence of DASH, a fibrillar coupler could facilitate a processive kinetochore-microtubule attachment in concert with the KMN network. As yet, the identity of the fibrous coupler is unknown. McIntosh et al. (2008) suggest that CENP-E could perform such a role. CENP-E is a kinetochore-associated kinesin that is essential for chromosome congression. EM reveals that CENP-E dimers form long $(\sim 230 \mathrm{~nm}$, similar to the longest observed fibrils) flexible fibres, due to a discontinuous coiled-coil separating its globular microtubule and kinetochore binding ends (Kim et al. 2008; McIntosh et al. 2008). A recent study has shown that Aurora B and PP1 cooperate to regulate CENP-E (Kim et al. 2010). Crucially, CENP-E is not conserved in yeast. As yet, however, there is no evidence that a CENP-E mediated interaction would allow processive microtubule tracking of the $\mathrm{Ndc} 80$ in the same way that DASH does.

Recently, the Ska complex was shown to share many properties with DASH and has been proposed 


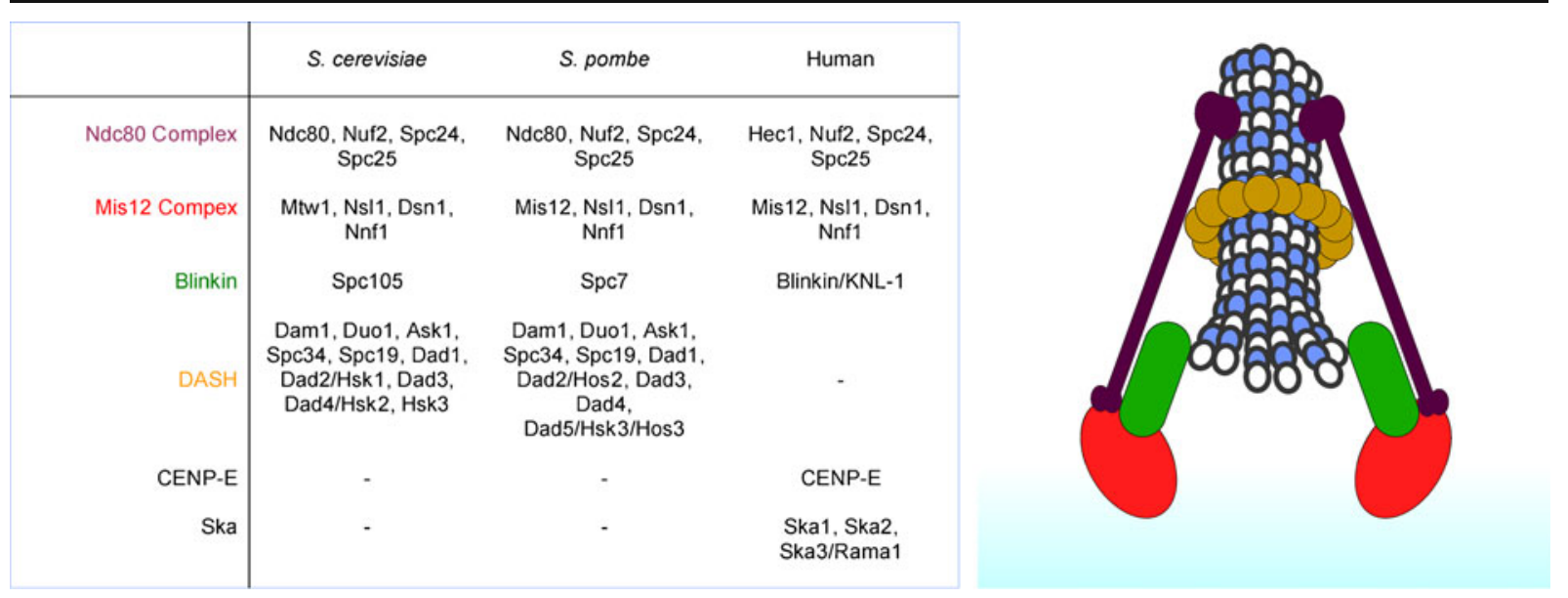

Fig. 3 Conservation of the core microtubule-binding site of the kinetochore between yeast and humans. Components of the KMN network are highly conserved; however, DASH is only

as a metazoan DASH analogue (Welburn et al. 2009). Accordingly, there are no Ska homologues in fungi (Fig. 3). Ska is a three-subunit outer kinetochore complex required for correct mitosis. All three subunits are required for Ska localisation and full activity (Gaitanos et al. 2009; Theis et al. 2009). Skadepleted cells show prolonged checkpoint-dependent mitotic arrest and mitotic defects (Hanisch et al. 2006; Gaitanos et al. 2009). Interestingly, Ska complex binds cooperatively to microtubules in vitro and, in a striking parallel to the DASH complex, forms ringlike assemblies around microtubules (Welburn et al. 2009). As with DASH, there is no evidence as yet that these in vitro rings represent a functional form of Ska in vivo. In addition, Ska complex-coated beads can undergo microtubule-linked movement of similar duration to those observed by DASH-coated beads (Asbury et al. 2006; Welburn et al. 2009).

The role of Ska during mitosis is still controversial. Some studies report weaker kinetochore-microtubule interactions in Ska-depleted cells, which may be consistent with a role analogous to DASH (Gaitanos et al. 2009); however, other studies detect robust kinetochore-microtubule interactions in Ska-depleted cells (Daum et al. 2009). In these studies, the main effect reported is prolonged spindle checkpoint delay coupled with loss of sister-chromatid cohesion. The extent to which the observed defects in Ska-depleted cells represent the absence of a DASH analogue remains to be seen. The key question to be answered is whether Ska can facilitate processive Ndc80 found in fungi. Schematic shows the major microtubulebinding components of the yeast kinetochore and their approximate relative location.

attachments to dynamic microtubules like DASH. Ska can only be considered a 'like-for-like' DASH analogue if the functional cooperation with $\mathrm{Ndc} 80$ can be recapitulated using the Ska complex.

In vitro studies of kinetochore-microtubule interactions have focused on interactions of kinetochore components with single microtubules (McIntosh et al. 2008; Akiyoshi et al. 2010; Lampert et al. 2010; Tien et al. 2010). This is a good model for S. cerevisiae kinetochores, which capture and track a single microtubule throughout mitosis. Given the dire consequences of failing to correctly segregate even a single chromosome, this represents a 'high stakes' mode of kinetochore capture. The essential role of the DASH complex may be to maintain kinetochore-microtubule attachment, as even a single detached kinetochore will lead to chromosome missegregation. S. pombe kinetochores attach to small bundles of two to four microtubules; therefore, DASH- deficient kinetochores may completely detach from the spindle less frequently than in S. cerevisiae. Accordingly, DASH is not essential in $S$. pombe, but DASH-deficient cells display mitotic checkpoint delay, a slower anaphase A and high rates of chromosome missegregation (Sanchez-Perez et al. 2005; Saitoh et al. 2008). Mammalian kinetochores attach to larger bundles of 20 or more microtubules, and in this case, a single detachment event will have little consequence, so a direct DASH analogue may be unnecessary. Interestingly, a recent study shows that the Ska complex is not essential for survival in DT40 cells (Ohta et al. 2010). 
Differences between fungal and metazoan $\mathrm{Ndc} 80$ complexes may account for the requirement for DASH at fungal kinetochores. Purified metazoan Ndc80 complexes bind cooperatively to microtubules in vitro (for a detailed review, see Tooley and Stukenberg, this issue) forming oligomeric 'clusters'. Cooperative microtubule binding requires the $\mathrm{N}$-terminus of the $\mathrm{Ndc} 80$ protein and is abrogated by mutations that mimic Aurora B phosphorylation (Alushin et al. 2010). The role of cooperative microtubule binding by $\mathrm{Ndc} 80$ has not been established. In their recent paper, Alushin et al. suggest that Ndc80 oligomers could track depolymerising microtubule ends by biased diffusion. Metazoan Ndc80 could therefore create a processive, Aurora B-regulated microtubule attachment in the absence of DASH. Presumably, bead-bound Ndc 80 complexes do not exhibit this cooperativity due to steric hinderance by the beads.

Whilst the N-terminus of $\mathrm{Ndc} 80$ is essential for kinetochore-microtubule interactions in human cells, mutations in the N-terminus of $S$. cerevisiae $\mathrm{Ndc} 80$ have comparatively mild phenotypes (Miller et al. 2008; Akiyoshi et al. 2009; Kemmler et al. 2009). Alushin et al. assert that the fungal Ndc80 complex has diverged to require DASH to enhance cooperativity. They identified a region close to the proposed $\mathrm{Ndc} 80$ oligomerisation site that is highly conserved in metazoans, but less so in fungi. They suggest that this site may be required for microtubule-dependent oligomerisation. In addition, they identified a second site that is conserved in fungi, but not metazoans. They propose this site as a candidate for the interaction of Ndc80 with DASH (Alushin et al. 2010).

If fungal $\mathrm{Ndc} 80$ complexes lack the capacity to self-organise, then the essential role of DASH in $S$. cerevisiae could be to organise the microtubule attachment site once sister chromatid biorientation has been achieved. This is consistent with the observation that DNA-tethered DASH is sufficient to organise a 'minimal' kinetochore competent for chromosome segregation (Kiermaier et al. 2009; Lacefield et al. 2009). Since $S$. pombe DASH is non-essential, this hypothesis implies that $S$. pombe Ndc80 complexes may have retained some limited capacity to self-organise beyond that of $S$. cerevisiae. Alternatively, there could be additional factors required to facilitate $\mathrm{Ndc} 80$ oligomerisation in these cells.

\section{Conclusion}

Recent insights into DASH function have brought a fuller understanding of the complex interactions between kinetochores and microtubules in yeast. In particular, a recent work suggests that DASH functions in concert with the Ndc80 complex to create a highly regulated, processive link between chromosomes and the spindle in fungi. Only by understanding how different kinetochore subunits act together in this way can we fully appreciate how the dynamic interface between the spindle and chromosomes acts to ensure chromosomal stability.

Despite the recent leaps made in our understanding of DASH function, there are still many questions left unanswered. For technical reasons, the individual roles of different DASH subunits are largely unresolved, and so it remains something of a mystery why DASH is made of so many small individual subunits. Similarly, DASH is essential in S. cerevisiae, but in the only other species with a well-studied DASH complex S. pombe, DASH appears to have a less prominent role. As $S$. pombe DASH has not been reconstituted in vitro, the extent to which this represents two functionally different versions of the same complex remains unclear.

Arguably, the major question raised by recent insights into DASH function is how non-fungal eukaryotes maintain regulated interactions between the kinetochore and microtubule in the absence of DASH. This review has mentioned two possible candidates for aiding this connection, although there are many more. A major focus of future studies will undoubtedly be to determine if any of the proposed candidates can fulfil this role in higher eukaryotes, or if fungal kinetochores are fundamentally diverged from those of other eukaryotes. Doing so will greatly enhance our understanding of how chromosomal stability is maintained in human cells.

Acknowledgments We thank John Meadows and Andrew McAinsh for critical reading of this manuscript.

\section{References}

Akiyoshi B, Nelson CR, Ranish JA, Biggins S (2009) Analysis of Ipl1-mediated phosphorylation of the Ndc 80 kinetochore protein in saccharomyces cerevisiae. Genetics 183(4):15911595 
Akiyoshi B, Sarangapani KK, Powers AF et al (2010) Tension directly stabilizes reconstituted kinetochore-microtubule attachments. Nature 468(7323):576-579

Alushin GM, Ramey VH, Pasqualato S et al (2010) The Ndc80 kinetochore complex forms oligomeric arrays along microtubules. Nature 467(7317):805-810

Armond JW, Turner MS (2010) Force transduction by the microtubule-bound Dam1 ring. Biophys J 98(8):15981607

Asbury CL, Gestaut DR, Powers AF, Franck AD, Davis TN (2006) The Daml kinetochore complex harnesses microtubule dynamics to produce force and movement. Proc Natl Acad Sci U S A 103(26):9873-9878

Biggins S, Severin FF, Bhalla N, Sassoon I, Hyman AA, Murray AW (1999a) The conserved protein kinase Ipl1 regulates microtubule binding to kinetochores in budding yeast. Genes Dev 13(5):532-544

Biggins S, Severin FF, Bhalla N, Sassoon I, Hyman AA, Murray AW (1999b) The conserved protein kinase Ipl1 regulates microtubule binding to kinetochores in budding yeast. Genes Dev 13(5):532-544

Cheeseman IM, Desai A (2008) Molecular architecture of the kinetochore-microtubule interface. Nat Rev Mol Cell Biol 9(1):33-46

Cheeseman IM, Brew C, Wolyniak M et al (2001a) Implication of a novel multiprotein Dam1p complex in outer kinetochore function. J Cell Biol 155(7):1137-1145

Cheeseman IM, Enquist-Newman M, Muller-Reichert T, Drubin DG, Barnes G (2001b) Mitotic spindle integrity and kinetochore function linked by the Duo1p/Dam1p complex. J Cell Biol 152(1):197-212

Cheeseman IM, Anderson S, Jwa M et al (2002) Phosphoregulation of kinetochore-microtubule attachments by the Aurora kinase Ipl1p. Cell 111(2):163-172

Cheeseman IM, Chappie JS, Wilson-Kubalek EM, Desai A (2006) The Conserved KMN network constitutes the core microtubule-binding site of the kinetochore. Cell 127 (5):983-997

Daum JR, Wren JD, Daniel JJ et al (2009) Ska3 is required for spindle checkpoint silencing and the maintenance of chromosome cohesion in mitosis. Curr Biol 19 (17):1467-1472

Dewar H, Tanaka K, Nasmyth K, Tanaka TU (2004) Tension between two kinetochores suffices for their bi-orientation on the mitotic spindle. Nature 428(6978):93-97

Ding R, McDonald KL, McIntosh JR (1993) Three-dimensional reconstruction and analysis of mitotic spindles from the yeast, Schizosaccharomyces pombe. J Cell Biol 120(1):141151

Efremov A, Grishchuk EL, McIntosh JR, Ataullakhanov FI (2007) In search of an optimal ring to couple microtubule depolymerization to processive chromosome motions. Proc Natl Acad Sci U S A 104(48):19017-19022

Enquist-Newman M, Cheeseman IM, Van Goor D, Drubin DG, Meluh PB, Barnes G (2001) Dad1p, third component of the Duolp/Dam1p complex involved in kinetochore function and mitotic spindle integrity. Mol Biol Cell 12 (9):2601-2613

Franck AD, Powers AF, Gestaut DR, Gonen T, Davis TN, Asbury CL (2007) Tension applied through the dam1 complex promotes microtubule elongation providing a direct mechanism for length control in mitosis. Nat Cell Biol 9(7):832-837

Franco A, Meadows JC, Millar JB (2007) The Dam1/DASH complex is required for the retrieval of unclustered kinetochores in fission yeast. J Cell Sci 120(Pt 19):33453351

Gachet Y, Reyes C, Courtheoux T et al (2008) Sister kinetochore recapture in fission yeast occurs by two distinct mechanisms, both requiring Dam1 and Klp2. Mol Biol Cell 19(4):1646-1662

Gaitanos TN, Santamaria A, Jeyaprakash AA, Wang B, Conti E, Nigg EA (2009) Stable kinetochore-microtubule interactions depend on the Ska complex and its new component Ska3/C13Orf3. EMBO J 28(10):1442-1452

Gao Q, Courtheoux T, Gachet Y, Tournier S, He X (2010) A non-ring-like form of the Dam1 complex modulates microtubule dynamics in fission yeast. Proc Natl Acad Sci USA 107(30):13330-13335

Gestaut DR, Graczyk B, Cooper J et al (2008) Phosphoregulation and depolymerization-driven movement of the Dam1 complex do not require ring formation. Nat Cell Biol 10(4):407-414

Griffiths K, Masuda H, Dhut S, Toda T (2008) Fission yeast dam1-A 8 mutant is resistant to and rescued by an antimicrotubule agent. Biochem Biophys Res Commun 368 (3):670-676

Grishchuk EL, McIntosh JR (2006) Microtubule depolymerization can drive poleward chromosome motion in fission yeast. EMBO J 25(20):4888-4896

Grishchuk EL, Spiridonov IS, Volkov VA et al (2008) Different assemblies of the DAM1 complex follow shortening microtubules by distinct mechanisms. Proc Natl Acad Sci USA 105(19):6918-6923

Hanisch A, Sillje HH, Nigg EA (2006) Timely anaphase onset requires a novel spindle and kinetochore complex comprising Ska1 and Ska2. EMBO J 25(23):5504-5515

Hofmann C, Cheeseman IM, Goode BL, McDonald KL, Barnes G, Drubin DG (1998) Saccharomyces cerevisiae Duolp and Dam1p, novel proteins involved in mitotic spindle function. J Cell Biol 143(4):1029-1040

Janke C, Ortiz J, Tanaka TU, Lechner J, Schiebel E (2002) Four new subunits of the Dam1-Duo1 complex reveal novel functions in sister kinetochore biorientation. EMBO J 21 (1-2):181-193

Joglekar AP, Bouck DC, Molk JN, Bloom KS, Salmon ED (2006) Molecular architecture of a kinetochore-microtubule attachment site. Nat Cell Biol 8(6):581-585

Joglekar AP, Bouck D, Finley K et al (2008) Molecular architecture of the kinetochore-microtubule attachment site is conserved between point and regional centromeres. J Cell Biol 181(4):587-594

Joglekar AP, Bloom K, Salmon ED (2009) In vivo protein architecture of the eukaryotic kinetochore with nanometer scale accuracy. Curr Biol 19(8):694-699

Jones MH, Bachant JB, Castillo AR, Giddings TH Jr, Winey M (1999) Yeast Dam1p is required to maintain spindle integrity during mitosis and interacts with the Mps1p kinase. Mol Biol Cell 10(7):2377-2391

Keating P, Rachidi N, Tanaka TU, Stark MJ (2009) Ipl1dependent phosphorylation of Dam 1 is reduced by tension applied on kinetochores. J Cell Sci 122(Pt 23):4375-4382 
Kemmler S, Stach M, Knapp M et al (2009) Mimicking Ndc80 phosphorylation triggers spindle assembly checkpoint signalling. EMBO J 28(8):1099-1110

Kiermaier E, Woehrer S, Peng Y, Mechtler K, Westermann S (2009) A Dam1-based artificial kinetochore is sufficient to promote chromosome segregation in budding yeast. Nat Cell Biol 11(9):1109-1115

Kim Y, Heuser JE, Waterman CM, Cleveland DW (2008) CENP-E combines a slow, processive motor and a flexible coiled coil to produce an essential motile kinetochore tether. J Cell Biol 181(3):411-419

Kim Y, Holland AJ, Lan W, Cleveland DW (2010) Aurora kinases and protein phosphatase 1 mediate chromosome congression through regulation of CENP-E. Cell 142 (3):444-455

Kobayashi Y, Saitoh S, Ogiyama Y, Soejima S, Takahashi K (2007) The fission yeast DASH complex is essential for satisfying the spindle assembly checkpoint induced by defects in the inner-kinetochore proteins. Genes Cells 12 (3):311-328

Lacefield S, Lau DT, Murray AW (2009) Recruiting a microtubule-binding complex to DNA directs chromosome segregation in budding yeast. Nat Cell Biol 11 (9):1116-1120

Lampert F, Hornung P, Westermann S (2010) The Dam1 complex confers microtubule plus end-tracking activity to the Ndc80 kinetochore complex. J Cell Biol 189(4):641649

Li Y, Elledge SJ (2003) The DASH complex component Ask1 is a cell cycle-regulated Cdk substrate in Saccharomyces cerevisiae. Cell Cycle 2(2):143-148

Li Y, Bachant J, Alcasabas AA, Wang Y, Qin J, Elledge SJ (2002) The mitotic spindle is required for loading of the DASH complex onto the kinetochore. Genes Dev 16 (2):183-197

Liu X, McLeod I, Anderson S, Yates JR 3rd, He X (2005) Molecular analysis of kinetochore architecture in fission yeast. EMBO J 24(16):2919-2930

Liu D, Vleugel M, Backer CB et al (2010) Regulated targeting of protein phosphatase 1 to the outer kinetochore by KNL1 opposes Aurora B kinase. J Cell Biol 188(6):809820

McAinsh AD, Tytell JD, Sorger PK (2003) Structure, function, and regulation of budding yeast kinetochores. Annu Rev Cell Dev Biol 19:519-539

McClelland SE, Burrell RA, Swanton C (2009) Chromosomal instability: a composite phenotype that influences sensitivity to chemotherapy. Cell Cycle 8(20):3262-3266

McIntosh JR (2005) Rings around kinetochore microtubules in yeast. Nat Struct Mol Biol 12(3):210-212

McIntosh JR, Grishchuk EL, Morphew MK et al (2008) Fibrils connect microtubule tips with kinetochores: a mechanism to couple tubulin dynamics to chromosome motion. Cell 135(2):322-333

Miller SA, Johnson ML, Stukenberg PT (2008) Kinetochore attachments require an interaction between unstructured tails on microtubules and $\mathrm{Ndc} 80(\mathrm{Hec} 1)$. Curr Biol 18 (22):1785-1791

Miranda JJ, De Wulf P, Sorger PK, Harrison SC (2005) The yeast DASH complex forms closed rings on microtubules. Nat Struct Mol Biol 12(2):138-143
Nicklas RB, Ward SC (1994) Elements of error correction in mitosis: microtubule capture, release, and tension. J Cell Biol 126(5):1241-1253

Nogales E, Ramey VH (2009) Structure-function insights into the yeast Dam1 kinetochore complex. J Cell Sci 122(Pt 21):3831-3836

Ohta S, Bukowski-Wills JC, Sanchez-Pulido L et al (2010) The protein composition of mitotic chromosomes determined using multiclassifier combinatorial proteomics. Cell 142 (5):810-821

Pinsky BA, Kotwaliwale CV, Tatsutani SY, Breed CA, Biggins S (2006a) Glc7/protein phosphatase 1 regulatory subunits can oppose the Ipl1/aurora protein kinase by redistributing Glc7. Mol Cell Biol 26(7):2648-2660

Pinsky BA, Kung C, Shokat KM, Biggins S (2006b) The Ipl1Aurora protein kinase activates the spindle checkpoint by creating unattached kinetochores. Nat Cell Biol 8(1):7883

Pinsky BA, Nelson CR, Biggins S (2009) Protein phosphatase 1 regulates exit from the spindle checkpoint in budding yeast. Curr Biol 19(14):1182-1187

Powers AF, Franck AD, Gestaut DR et al (2009) The Ndc80 kinetochore complex forms load-bearing attachments to dynamic microtubule tips via biased diffusion. Cell 136 (5):865-875

Przewloka MR, Glover DM (2009) The kinetochore and the centromere: a working long distance relationship. Annu Rev Genet 43:439-465

Przewloka MR, Zhang W, Costa P et al (2007) Molecular analysis of core kinetochore composition and assembly in Drosophila melanogaster. PLoS ONE 2(5):e478

Rieder CL, Alexander SP (1990) Kinetochores are transported poleward along a single astral microtubule during chromosome attachment to the spindle in newt lung cells. J Cell Biol 110(1):81-95

Saitoh S, Kobayashi Y, Ogiyama Y, Takahashi K (2008) Dual regulation of Mad2 localization on kinetochores by Bub1 and Dam1/DASH that ensure proper spindle interaction. Mol Biol Cell 19(9):3885-3897

Salmon ED (2005) Microtubules: a ring for the depolymerization motor. Curr Biol 15(8):R299-R302

Sanchez-Perez I, Renwick SJ, Crawley K et al (2005) The DASH complex and Klp5/Klp6 kinesin coordinate bipolar chromosome attachment in fission yeast. EMBO J 24 (16):2931-2943

Sawin KE, Lourenco PC, Snaith HA (2004) Microtubule nucleation at non-spindle pole body microtubule-organizing centers requires fission yeast centrosomin-related protein $\bmod 20$ p. Curr Biol 14(9):763-775

Shang C, Hazbun TR, Cheeseman IM et al (2003) Kinetochore protein interactions and their regulation by the Aurora kinase Ipl1p. Mol Biol Cell 14(8):3342-3355

Shimogawa MM, Graczyk B, Gardner MK et al (2006) Mps1 phosphorylation of Dam1 couples kinetochores to microtubule plus ends at metaphase. Curr Biol 16(15):1489-1501

Tanaka TU, Desai A (2008) Kinetochore-microtubule interactions: the means to the end. Curr Opin Cell Biol 20 (1):53-63

Tanaka K, Mukae N, Dewar H et al (2005) Molecular mechanisms of kinetochore capture by spindle microtubules. Nature 434 (7036):987-994 
Tanaka K, Kitamura E, Kitamura Y, Tanaka TU (2007) Molecular mechanisms of microtubule-dependent kinetochore transport toward spindle poles. J Cell Biol 178(2):269-281

Theis M, Slabicki M, Junqueira M et al (2009) Comparative profiling identifies C13orf3 as a component of the Ska complex required for mammalian cell division. EMBO J 28(10):1453-1465

Thompson SL, Bakhoum SF, Compton DA (2010) Mechanisms of chromosomal instability. Curr Biol 20(6):R285-R295

Tien JF, Umbreit NT, Gestaut DR et al (2010) Cooperation of the Dam1 and Ndc80 kinetochore complexes enhances microtubule coupling and is regulated by aurora B. J Cell Biol 189(4):713-723

Vanoosthuyse V, Hardwick KG (2009) A novel protein phosphatase 1-dependent spindle checkpoint silencing mechanism. Curr Biol 19(14):1176-1181

Vanoosthuyse V, Meadows JC, van der Sar SJ, Millar JB, Hardwick KG (2009) Bub3p facilitates spindle checkpoint silencing in fission yeast. Mol Biol Cell 20(24):5096-5105

Waldo J, Scherrer M (2008) Production and initial characterization of Dad1p, a component of the Dam1-DASH kinetochore complex. PLoS ONE 3(12):e3888
Wang HW, Ramey VH, Westermann S et al (2007) Architecture of the Dam1 kinetochore ring complex and implications for microtubule-driven assembly and force-coupling mechanisms. Nat Struct Mol Biol 14(8):721-726

Weiss E, Winey M (1996) The Saccharomyces cerevisiae spindle pole body duplication gene MPS1 is part of a mitotic checkpoint. J Cell Biol 132(1-2):111-123

Welburn JP, Grishchuk EL, Backer CB, Wilson-Kubalek EM, Yates JR 3rd, Cheeseman IM (2009) The human kinetochore Ska1 complex facilitates microtubule depolymerizationcoupled motility. Dev Cell 16(3):374-385

Westermann S, Avila-Sakar A, Wang HW et al (2005) Formation of a dynamic kinetochore- microtubule interface through assembly of the Dam1 ring complex. Mol Cell 17(2):277-290

Westermann S, Wang HW, Avila-Sakar A, Drubin DG, Nogales E, Barnes G (2006) The Dam1 kinetochore ring complex moves processively on depolymerizing microtubule ends. Nature 440(7083):565-569

Wilson-Grady JT, Villen J, Gygi SP (2008) Phosphoproteome analysis of fission yeast. J Proteome Res 7 (3):1088-1097 